\title{
Einschüchtern und Vertreiben, Bestrafen und Eskalieren. Instrumentelle Zielsetzungen rechtsterroristischen Handelns
}

\author{
Fabian Virchow
}

Eingegangen: 4. August 2021 / Angenommen: 4. Oktober 2021 / Online publiziert: 6. Dezember 2021 (C) Der/die Autor(en) 2021

Zusammenfassung Der Beitrag entwickelt anhand zahlreicher Beispiele rechtsterroristischer Gewalt eine Systematisierung instrumenteller Zielsetzungen rechtsterroristischen Handelns. Dabei ruft er zunächst als zentrale weltanschauliche Referenz den völkischen Nationalismus auf, dem biopolitische Paradigmen inhärent sind, denen mittels rechtsterroristischer Gewalt Geltung verschafft werden soll. Dieses ist für eine Vielzahl rechtsterroristischer Gewalttaten zentral, denen es um die Aufrechterhaltung rassistischer, sexistischer und heteronormativer Gesellschaftsverhältnisse geht. Regelmäßig ist solche Gewalt auch als Botschaftsverbrechen zu verstehen, wobei ganz unterschiedliche Modi der Kommunikation beobachtbar sind. Rechtsterroristische Gewalt - sofern sie nicht parastaatlich verfasst ist - hat nicht die direkte Schwächung der Kampffähigkeit des Gegners zum Ziel, sondern zielt auf Bestrafung, Einschüchterung und Vertreibung ausgewählter sozialer Gruppen und Individuen sowie in manchen Fällen auf das strategische Moment der Zuspitzung gesellschaftlicher Konflikte, wie im abschließenden Teil des Beitrages an zahlreichen Beispielen gezeigt wird.

Schlüsselwörter Rechtsterrorismus · Instrumentelle Zielsetzung · Gewaltsoziologie · Biopolitik · Politisches Handeln · Rechtsextremismus

Fabian Virchow $(\bowtie)$

Fachbereich Sozial- und Kulturwissenschaften, Hochschule Düsseldorf, Düsseldorf, Deutschland E-Mail: fabian.virchow@hs-duesseldorf.de 


\title{
Intimidation and Expulsion, Punishment and Escalation. Instrumental Objectives of Right-Wing Terrorist Action
}

\begin{abstract}
On the basis of numerous examples of right-wing terrorist violence, the article develops a systematization of instrumental objectives of right-wing terrorist actions. In doing so, the article first calls up völkisch nationalism as a central ideological reference, which has inherent biopolitical paradigms that are to be enforced by means of right-wing terrorist violence. This is central to a large number of rightwing terrorist acts of violence, which are aimed at maintaining racist, sexist and heteronormative social relations. As a rule, such violence is also to be understood as message crime, whereby quite different modes of communication can be observed. Right-wing terrorist violence — as long as it is not parastatal-does not aim at directly weakening the opponent's military ability to fight, but rather at punishing, intimidating and expelling selected social groups and individuals, as well as in some cases at the strategic moment of intensifying social conflicts, as will be shown in the concluding part of the article with several examples.
\end{abstract}

Keywords Right-wing terrorism · Instrumental goal setting · Sociology of violence $\cdot$ Biopolitics $\cdot$ Political action $\cdot$ Right-wing extremism

\section{Perspektiven auf Terrorismus}

Soziologische Forschung zu Terrorismus hat sich entlang ganz unterschiedlicher Fragestellungen entwickelt, die vielfach enge Bezüge zu einer Soziologie sozialer Probleme bzw. einer Soziologie der sozialen Kontrolle aufweisen; hierzu gehörten insbesondere die Untersuchung des sozialen Konstruktionscharakters von Terrorismus, seiner Spezifik als politisch motivierte Gewalt, des Terrorismus als kommunikativer Akt, terroristischer Organisationsformen und Handlungspraxen, der Sozialisationsprozesse ihrer Akteur:innen sowie schließlich Fragen nach der sozialen Kontrolle terroristischer Gewalt (Turk 2004). Angesichts der Vielzahl von erkenntnistheoretischen wie disziplinären Zugängen in der Terrorismusforschung hat Dunning (2021) mit Blick auf eine mögliche Systematisierung ein Kontinuum vorgeschlagen, ,that has at one end variants of logical positivism and realism, and at the other, approaches that are more closely associated with epistemic relativism, such as various forms of social constructivism“ (Dunning 2021, S. 14). Dabei verortet er die erstgenannten Ansätze in größerer Nähe zu staatlichen Instanzen und deren auf Terrorismus bezogenen Perspektiven und Handlungspraxen, während letztere stärker hervorheben, wie Terrorismus als Problem sozial konstruiert wird und wie in solchen Konstruktionsprozessen auch Machtverhältnisse zum Ausdruck kommen. Mit Blick auf die Geschichte des Rechtsterrorismus in Deutschland haben die in Folge des Bekanntwerdens der rassistischen Morde des Nationalsozialistischen Untergrunds (NSU) in mehreren Bundesländern beschlossenen parlamentarischen Untersuchungsausschüsse bereits zahlreiche Hinweise und Beispiele zu Tage fördern können, dass und wie Gewalt von rechts in Diskursen medialer, politischer und staatlicher Protagonist:innen eben nicht als rechte Gewalt bzw. Rechtsterrorismus bezeichnet und ein- 
geordnet wurde. Eine umfassende Diskursgeschichte zum Rechtsterrorismus steht allerdings trotz vereinzelter Vorarbeiten (als exemplarische Fallstudie: Virchow et al. 2015) noch aus.

Zu Recht merkt Dunning (2021, S. 15) an, dass eine rein sozialkonstruktivistische Perspektive die tatsächlichen Folgen des Handelns terroristischer Akteur:innen, aber auch - mit Norbert Elias sprechend - die figurative Dynamik, als deren Ergebnis bestimmte Gruppen (nicht) als Terrorist:innen bezeichnet werden, nicht zu fassen vermag. Die Geschichte Deutschlands im 20. Jahrhundert liefert für eine entsprechende prozesssoziologische Perspektive vielseitige Anhaltspunkte, die hier freilich nicht ausgearbeitet werden können. Es sei lediglich darauf verwiesen, dass Rechtsterrorismus in der Weimarer Republik mit seinen antisemitischen, antikommunistischen und biopolitischen Motivlagen angesichts starker republik- und demokratiefeindlicher Akteursgruppen in der zeitgenössischen juristischen Aufarbeitung wie politischen Bewertung auf Verständnis, wenn nicht vielfach auch auf Zustimmung traf. Entsprechendes Handeln wurde dann mit der Machtübernahme der nazistischen Bewegung und der damit verbundenen Möglichkeit der Mobilisierung des gesamten Staatsapparates zur Realisierung einer rassenideologischen Reinheitsund Expansionspolitik idolisiert, um nach der Niederlage des deutschen Faschismus in den beiden deutschen Nachfolgegesellschaften diskursiv weitgehend verdrängt, beschwiegen oder externalisiert zu werden (Virchow und Thomas 2021).

In das erste Jahrzehnt des 21. Jahrhunderts fällt die rassistische Mordserie des $N S U$ als ein bis dahin weithin unbekannter Modus der Umsetzung rechtsterroristischen Handelns in der Bundesrepublik Deutschland in Gestalt einer langjährig ,klandestin ' operierenden Zelle, die ihre Opfer aus nächster Nähe tötete. ${ }^{1}$ Die vergangenen zehn Jahre (2010-2020) markieren in verschiedener Hinsicht eine neue Phase der Entwicklung des Rechtsterrorismus und seiner Wahrnehmung in Deutschland. Diese Zeitspanne ist erstens dadurch charakterisiert, dass aufgrund der Morde des NSU einer breiten Bevölkerungsmehrheit die Existenz rechtsterroristischer Strukturen vor Augen geführt wurde; zweitens ist rechtsterroristische Gewalt ausgeweitet worden auf hochrangige Vertreter:innen des Staates, die sich für eine liberale Migrationspolitik eingesetzt haben (Steinhagen 2021); drittens sind zahlreiche weitere rechtsterroristische Aktivitäten aufgeflogen und strafrechtlich abgeurteilt worden (Philippsberg 2021); viertens hat auch die Perspektive von Betroffenen und Überlebenden rechter Gewalt aufgrund aktivistischer Mobilisierung größere Sichtbarkeit erhalten (z. B. Şimşek 2013; John 2014; Bozay et al. 2016, 2021). Und schließlich hat sich die wissenschaftliche Befassung mit Rechtsterrorismus im deutschsprachigen Kontext intensiviert (z.B. Koehler 2016; Virchow 2016; Gräfe 2017; Schultz 2018; Quent 2019; Lüttig und Lehmann 2020).

Die Frage, welche empirischen Phänomene mit dem Begriff (Rechts)Terrorismus belegt werden sollen, ist nicht abschließend beantwortet und soll auch nicht Gegenstand dieses Beitrages sein. Mit Hess (1988a, S. 59) ließe sich Terrorismus verstehen als ,erstens eine Reihe von vorsätzlichen Akten direkter, physischer Gewalt, die zweitens punktuell und unvorhersehbar, aber systematisch drittens mit dem

\footnotetext{
${ }^{1}$ Dass auch klandestin tätige politische Gruppen nur aufgrund ihrer Einbindung in Unterstützungsnetzwerke dauerhaft existieren können, ist evident (Della Porta 2013).
} 
Ziel psychischer Wirkung auf andere als das physisch getroffene Opfer viertens im Rahmen einer politischen Strategie ausgeführt werden“. Hilfreich ist mit Blick auf den Rechtsterrorismus zudem die Definition von Manthe (2020, S. 68), die diesen versteht als

das geplante Agieren von Gruppen oder Einzelpersonen ..., die aufgrund einer extrem rechten Weltanschauung beziehungsweise einer Einbindung in ein extrem rechtes Milieu konspirativ und unter versuchter oder tatsächlicher Anwendung von schwerer Gewalt gegen Personen oder Objekte mindestens zwei der folgenden Ziele verfolgen: a) ein Klima der Angst in der Bevölkerung oder bei bestimmten Bevölkerungsteilen zu erzeugen, b) Aufmerksamkeit zu erregen/ Öffentlichkeit herzustellen, c) staatliche, gesellschaftliche oder politische Akteure in ihrem Handeln zu beeinflussen/eine Überreaktion durch die Behörden zu provozieren, d) die politische oder gesellschaftliche Ordnung zu destabilisieren oder zu verteidigen (Vigilantismus).

Im Folgenden soll es nicht um eine chronologische Darstellung rechtsterroristischer Gewalt gehen, sondern der Versuch einer Systematisierung unternommen werden, die anhand zahlreicher Beispiele verdeutlicht, welchen instrumentellen Zielsetzungen rechtsterroristische Taten folgen können. Unter instrumentellen Zielsetzungen sollen hier die in Entscheidungssituationen gewählten Mittel und Handlungen zum Erreichen von Zielen (als Etappen in einem längeren Entwicklungsprozess) verstanden werden. Fischer (2014, S. 192f.) nennt als typische strategische bzw. instrumentelle Zielsetzungen terroristischer Gruppierungen

das Aufstellen von Forderungen und die Drohung mit weiterer Gewalt für den Fall der Nichterfüllung ...; die Mobilisierung der Bezugsgruppe zum Widerstand - sei es dadurch, dass die Möglichkeit des Widerstands demonstriert wird, sei es durch die Provokation staatlicher Repression, die widerstandsfördernd wirken soll; die Erzeugung von Aufmerksamkeit für ein ansonsten weniger beachtetes Problem ... und die Unterminierung des staatlichen Legitimitätsanspruchs - durch die Demonstration, dass der Staat seine Bürger nicht schützen kann.

Mit Wojciechowski (2017, S. 51) ließe sich noch die ,revenge for a certain activity of government or its representatives" nennen. Mit Blick auf rechtsterroristische Gewalt - vornehmlich in Gesellschaften des globalen Nordens - ruft der Beitrag zunächst (2) als zentrale weltanschauliche Referenz den völkischen Nationalismus auf, dem biopolitische Paradigmen inhärent sind, denen mittels rechtsterroristischer Gewalt Geltung verschafft werden soll. Dieses Paradigma ist für eine Vielzahl rechtsterroristischer Gewalttaten zentral, wie sich anhand von Beispielen zeigen lässt, denen es um die Aufrechterhaltung rassistischer, sexistischer und heteronormativer Gesellschaftsverhältnisse geht. Dass solche Taten regelmäßig auch als Botschaftsverbrechen zu verstehen sind, wird im folgenden Abschnitt (3) unter Berücksichtigung ganz unterschiedlicher Modi der Kommunikation exemplarisch diskutiert und veranschaulicht. Diese Botschaftsgewalt richtet sich vielfach auch gegen die politische und gewerkschaftliche Linke. Rechtsterroristische Gewalt - sofern sie nicht parastaatlich verfasst ist - hat nicht die direkte Schwächung der Kampf- 
fähigkeit des Gegners zum Ziel, sondern zielt auf Bestrafung, Einschüchterung und Vertreibung ausgewählter sozialer Gruppen und Individuen (4) sowie in manchen Fällen auf das strategische Moment der Zuspitzung gesellschaftlicher Konflikte, wie im abschließenden Teil des Beitrages (5) an zahlreichen Beispielen gezeigt wird.

\section{Rechtsterrorismus als bio-politische Gewaltpraxis}

Thomas Lemke (2008) identifiziert drei Lesarten des Begriffs Biopolitik: Neben einem naturalistischen Verständnis, das auf eine behauptete Naturbasis der Politik abhebt, das zu Beginn des 20. Jahrhunderts beispielsweise in organizistischen Staatskonzepten wie demjenigen Rudolf Kjelléns (1920) sichtbar geworden sei und seit Mitte der 1960er-Jahre auch als politikwissenschaftlicher Ansatz an Hochschulen auftrete (Peterson und Somit 2017), existiere eine politizistische Lesart, der es in jüngerer Zeit vor allem um Fragen rund um die ,kollektive Aushandlung und Bewertung der sozialen und politischen Implikationen neuer medizinischer Technologien und biowissenschaftlicher Innovationen“ (Lemke 2008, S. 77 ff.) gehe. Mit den archäologisch-genealogischen Untersuchungen Michel Foucaults (2004) liege schließlich ein dritter - relationaler - Begriff vor, bei dem Bio-Politik für eine Konstellation stehe, , in der die modernen Human- und Naturwissenschaften und die aus ihnen hervorgehenden Normalitätskonzepte das politische Handeln strukturieren und dessen Ziele bestimmen“, so dass sich darin eine ,fundamentale Veränderung in der Ordnung des Politischen“ (Lemke 2008, S. 80) ausdrücke. Mit dem Auftreten des Liberalismus als Regierungsform und der Entwicklung neuer wissenschaftlicher Disziplinen wie Statistik, Demografie, Epidemiologie und Biologie rückte in den Mittelpunkt, „die Lebensprozesse auf dem Niveau von Bevölkerungen [zu] analysieren, um Individuen und Kollektive mittels korrigierender, exkludierender, normalisierender, disziplinierender, therapierender oder optimierender Maßnahmen zu ,regieren“" (ebd., S. 81). Die hierbei machtvoll wirkenden Wissensbestände sind historisch veränderlich und setzen je spezifische Normalitätsvorstellungen relevant. Entsprechend lässt sich m. E. eine liberale von einer faschistischen Biopolitik unterscheiden, wobei letztere völkische Homogenitätsideale und heteronormative Geschlechterarrangements als normative Bezugspunkte der Fremd- und Selbstführung aufruft und ggfs. mit Gewalt durchsetzen möchte. Nicht zufällig wurde der Terminus ,Biopolitik' seit den frühen 1930er-Jahren vereinzelt explizit von NS-Bevölkerungsplanern verwendet (Sarasin und Thomä 2008, S. 147).

Zwar geht Foucault davon aus, dass die Biopolitik - anders als noch vorhergehende archaische Formen des Regierens, die sich vor allem auf die unbeschränkte Drohung mit Gewalt und Tod stützten - ,eher auf der Verwaltung des Lebens als auf der Drohung mit dem Tode beruht“ (Foucault 1983, S. 142) und der ,,vollständigen Durchsetzung des Lebens gilt“" (ebd., S. 167); angesichts der zentralen Bedeutung, die Foucault dem Rassismus als eine wesentliche ideologische Grundlage moderner Staatsmacht zuweist, kann es jedoch kaum verwundern, dass er im NS-Regime ,wohl tatsächlich die auf die Spitze getriebene Entwicklung neuer, seit dem 18. Jahrhundert vorhandener Machtmechanismen“ sah: 
Es gibt keinen disziplinäreren Staat als das Naziregime; auch keinen Staat, in dem die biologischen Regulierungen auf straffere und nachdrücklichere Weise wiederaufgenommen worden wären. Disziplinarmacht, Bio-Macht: Beide hat die Nazigesellschaft mitaufgenommen und zum Einsatz gebracht (die Erfassung des Biologischen, der Fortpflanzung, der Erbschaft; Erfassung auch von Krankheit und Unfällen). Keine Gesellschaft, die disziplinärer und zugleich absichernder gewesen wäre als die von den Nazis eingeführte oder in jedem Fall geplante. Die Kontrolle der den biologischen Prozessen eigenen Zufälle war eines der unmittelbaren Ziele dieses Regimes. (Foucault 1999, S. 306)

Die gegenwärtige Weltanschauung der extremen Rechten kennt entsprechend zahlreiche Anrufungen der Selbstführung im Sinne der Erhaltung von ,Volk` und ,Volksgesundheit" wie rassistische und antisemitische Begründungen der Exklusion (Bott 1969; Pörksen 2000). Zur Bandbreite ihrer Handlungspraxen zählt die gewaltförmige Durchsetzung entsprechender biopolitischer Ziele. Hierunter fallen rechtsterroristische Akte der Gewalt gegen migrantisierte Teile der Bevölkerung, gegen Ärtz:innen, die Schwangerschaftsabbrüche durchführen, aber auch gegen selbstbewusst ausgelebtes Begehren jenseits der heteronormativen Matrix.

Der angestrebten biopolitischen Zielsetzung eines völkischen Deutschland in einem weißen Europa folgend, zielt extrem rechte Politik neben der Unterbindung weiterer Einwanderung auf die „Rückführung der Ausländer in ihre Heimat“ (NPD 2015). Dabei soll eine gesetzlich fixierte Diskriminierung, etwa hinsichtlich des Zugangs zu Sozialleistungen oder des Rechts auf Erwerb von Grund und Boden, diese Vertreibungspolitik voranbringen. Teil einer langen Tradition rassistischer Gewalt, bei der in den beiden deutschen Staaten seit 1949 Tausende schwer verletzt und über 150 Menschen getötet wurden (Billstein 2020, S. 22), sind auch entsprechende rechtsterroristische Gewaltakte.

Nur exemplarisch können hier genannt werden die 1980 von den Deutschen Aktionsgruppen (DA) um Manfred Roeder (Strohmaier 1982) innerhalb von drei Wochen verübten Brandanschläge auf Unterkünfte für Asylsuchende in Zirndorf, LeinfeldenEchterdingen, Lörrach und Hamburg, bei dem am 20. August 1980 Nguyễn Ngọc Châu und Đô̂ Anh Lân ihr Leben verloren. 1982 tötete der Nürnberger Neonazi Helmut Oxner durch Waffengewalt drei Menschen, die er für Nicht-Deutsche hielt, und verletzte weitere Personen schwer. Zwischen 2003 und 2005 setzten die Mitglieder des Freikorps Havelland aufgrund ihres anti-asiatischen Rassismus in mehreren Ortschaften Brandenburgs Imbisswagen, Restaurants oder Geschäfte in Brand, um die Eigner:innen zu vertreiben. Der $N S U$ zielte mit seinen Bombenanschlägen und Morden auf die Vernichtung migrantischen Lebens in Deutschland. Die Zunahme der Zahl von Schutzsuchenden Mitte der 2010er-Jahre wurde von Rassist:innen mit weiteren Gewalttaten beantwortet; bei den in der Folgezeit entstehenden rechtsterroristischen Gruppen Oldschool Society, Gruppe Freital sowie Revolution Chemnitz. ist die rassistische Motivation, die zum Teil eliminatorische Züge aufweist, ein zentrales Motiv (Philippsberg 2021, S. 158). Die Bio-Politik, so Foucault, führt mit dem Rassismus einen Modus der signifikanten hierarchisierenden Distinktion ein, die markiert, welches Leben existieren darf und welches (zur Erfüllung dieses Zweckes) zu verschwinden hat. 
Auch die auf das Begehren und die Herstellung der heteronormativen Geschlechterordnung gerichtete rechtsterroristische Gewalt blickt auf eine lange Linie zurück; der Mordanschlag auf den Sexualwissenschaftler Magnus Hirschfeld am 4. Oktober 1920 mag hier als Referenz ausreichen (Knoll 2020). Zwischen 1977 und 1984 verübte die Gruppe Ludwig mindestens zehn Anschläge in Deutschland und Norditalien. Die Morde richteten sich insbesondere gegen Homosexuelle und Drogenabhängige; bei einem Brandanschlag auf das Pornokino Eros in Mailand kamen am 14. Mai 1983 sechs Männer um (Caneva 1986; Zornetta 2011). An drei aufeinander folgenden Wochenenden brachte David Copeland, zuvor Mitglied der British National Party und des National Socialist Movement, Mitte April 1999 in London Nagelbomben zur Explosion - zunächst in dem vor allem von Schwarzen bewohnten Stadtteil Brixton, dann im East End, einem Zentrum bengalischer Einwanderung, sowie in einem Pub in Soho, wo sich die homosexuelle und queere Szene zum Feiern am Wochenende traf. Die auf bio-politische ,Reinigung ‘ zielenden Gewaltakte forderten insgesamt drei Tote und 150 Verletzte.

Schließlich ist als rechtsterroristische Bio-Politik auch die organisierte Gewalt gegen Ärtz:innen und gegen Kliniken zu nennen - beispielsweise in den USA -, in denen Schwangerschaftsabbrüche vorgenommen werden (Baird-Windle und Bader 2001; Wilson 2013). Deren Ziel ist es, durch (die Drohung mit) Gewalt ärztliches Personal einzuschüchtern und von der Durchführung von Schwangerschaftsabbrüchen abzuhalten, um auf diesem Wege das Selbstbestimmungsrecht der Frauen (pro choice) zu untergraben. Insbesondere seit den frühen 1990er-Jahren hat diese Gewalt in Form von Schusswaffengebrauch, Brand- und Bombenanschlägen erheblich zugenommen.

\section{Rechtsterrorismus als Botschaftsverbrechen}

In der Terrorismusforschung ist im Grundsatz unbestritten, dass Terrorismus auch als Akt der Kommunikation verstanden werden kann (Schmid und de Graaf 1982; Waldmann 2005, S. 33-46; Nossek 2008; Tuman 2010). Dies beantwortet allerdings noch nicht die Frage, welchen Stellenwert diese Dimension hat, wie sie angemessen zu untersuchen ist - etwa als Diskursanalyse oder mit Blick auf die Rezeption -, in welchen Formen diese Kommunikation empirisch anzutreffen ist und ob bzw. wie sich der Modus der Kommunikation im Zuge der Digitalisierung substantiell verändert hat (Post et al. 2014).

Aus kommunikationswissenschaftlicher Perspektive erzwingt die im terroristischen Akt zum Ausdruck kommende ,(gewaltbehaftete) Beendigung von Kommunikation ... demnach Anschlusskommunikation“ (Rothenberger 2020, S. 330) - insbesondere, aber nicht ausschließlich in Medien. Die von Rechtsterrorist:innen Angegriffenen und Getöteten sind - folgt man Fuchs (2004, S. 23) - „Doppelopfer: als getötete, verstümmelte Körper und dadurch, dass sie für die Dramaturgie der Mitteilung instrumentalisiert werden“. Die Schlussfolgerung, dass es daher egal sei, „wer stirbt, da nicht bestimmte Individuen [sterben], sondern nur Körper in ihrer Funktion als Personen zählen“ (Rothenberger 2020, S. 331), übersieht jedoch, dass zahlreiche rechtsterroristische Akte - den Feindbildkonstruktionen seiner Weltdeu- 
tung folgend - sehr bewusst die (Gruppe der) Angegriffenen und immer wieder auch konkrete Individuen als Ziel auswählen. So ist, schussfolgerte Hess schon früh, die Violenz des Terrorismus kommunikativ (Hess 1988a, S. 60).

Mit Blick auf (rechts)terroristische Gewalt lässt sich eine erhebliche Bandbreite der Kommunikationsmodi feststellen. Diese reichen vom Verzicht auf ein explizites Bekenntnis und die Erläuterung der Tatmotive über knappe verbale oder schriftliche Bekenntnisse (Unterholzner 2007) hin zu umfangreichen Manifesten, wie sie beispielsweise Anders Behring Breivik verfasst und verbreitet hat (Jackson 2013; Sandberg 2013; Gullestad 2017). In Bekenntnissen legitimieren die Täter:innen das

Verletzen der Normen für einen „höheren Zweck“, nämlich, die öffentliche Aufmerksamkeit auf ein dringliches Problem zu lenken. Zu dieser Rechtfertigungsstrategie gehört zudem, der Fremdattribution ,Terrorist“ entgegenzutreten und die eigene Gruppe mit eher positiv konnotierten Begriffen wie „Freiheitskämpfer“ oder „Revolutionäre“ zu belegen. Die Gruppe versucht damit, ihre Aktivitäten und Grenzübertritte oder Normverstöße zu legitimieren (Rothenberger 2020, S. 17).

Viele rechtsterroristische Gewaltakte bleiben ohne explizites Bekenntnis, so etwa der Sprengstoffanschlag auf das Grab des vormaligen Vorsitzenden des Zentralrats der Juden in Deutschland, Heinz Galinski, im Herbst 1998 oder die Ermordung des Kasseler Regierungspräsidenten Walther Lübcke Anfang Juni 2019. Ein solches Vorgehen kann - so wird in Publikationen aus dem Spektrum der extremen Rechten wie den Turner Diaries (Wilson 2014) nahegelegt - mit Blick auf mögliche Fahndungsansätze gewählt werden, aber auch der Überlegung geschuldet sein, dass die Auswahl der Opfer bereits ,für sich' spräche. Entsprechend betont Gräfe (2017) in seiner Untersuchung zum Rechtsterrorismus in Deutschland wiederholt, dass die Auswahl der Opfer unmittelbar auf das Motiv verweise: „Die Anschläge auf Asylbewerberunterkünfte und Ausländerheime erklärten sich für die Gruppe [gemeint sind hier die Deutschen Aktionsgruppen, F. V.] wahrscheinlich von selbst, da Anschlagsziel und Opfer identisch waren“ (Gräfe 2017, S. 141). Auch der Nationalsozialistische Untergrund vertraute auf die Signalwirkung seiner Taten; entgegen einer dominanzgesellschaftlichen Lesart, dass man nicht habe wissen können, dass einer Mordserie an migrantisierten Menschen rassistische Motive zugrunde liegen, muss konstatiert werden, dass die durch die Morde vermittelte Botschaft sowohl von der neonazistischen Szene wie bei den Betroffenen erkannt und kommuniziert wurde. Bei den einen beispielweise als Verherrlichung der Taten und in Form expliziter Thematisierung der mit ihnen verbundenen Effekte von Angst und Schrecken im Lied „Döner-Killer“ der neonazistischen Band „Gigi \& die braunen Stadtmusikanten“, bei den anderen gegenüber den Ermittlungsbehörden.

Wenig elaborierte Formen des Bekenntnisses sind das Aufhängen einschlägig konnotierter Flaggen (Brown 2020), gesprühte Hakenkreuze oder SS-Runen als Teil der Zerstörung jüdischer Grabstätten (Steinke 2020) oder das mit Schweineblut aufgebrachte Kürzel ,C18‘ (=Combat 18) an einer Gedenktafel für KZ-Häftlinge in Neustadt/Holstein (Landtag Nordrhein-Westfalen 2017, S. 191). Knappe telefonische Bekenntnisse finden sich ebenfalls. Die Deutschen Aktionsgruppen bekannten sich bei den frühen Anschlägen telefonisch oder setzten Zeitungsredaktionen in 
Kenntnis (Gräfe 2017, S. 140, unter Bezug zum Urteil des OLG Stuttgart); der englische Rechtsterrorist David Copeland nutzte im Zusammenhang mit seinem Bombenanschlag am 24. April 1999, der sich insbesondere gegen Einwander:innen aus Bangladesh richtete, ein öffentliches Tastentelefon und setzte ein Bekenntnis als Combat 18 ab (Landtag Nordrhein-Westfalen 2017, S. 173). Zu der von Copeland verübten rassistischen und homosexuellenfeindlichen Anschlagsserie in London hatten sich unabhängig voneinander zunächst vier neonazistische Gruppen bekannt (Kearns et al. 2014, S. 431), bevor David Copeland als Täter identifiziert und verurteilt wurde.

Falsche Bekenntnisse (false flag) sind für terroristisches Handeln nicht ungewöhnlich und werden von verschiedenen Motiven getragen (Jenkins 1988), darunter auch dem Versuch, den politischen Gegner zu diskreditieren oder durch gezielte Hinweise den Verdacht auf diesen zu lenken. In Frankreich verübte die neonazistische Organisation Parti nationaliste français et européen (PNFE) Ende 1988 mehrere Anschläge auf Unterkünfte arabischer Arbeitsmigrant:innen und hinterließ Flugblätter, in denen sich eine vermeintlich jüdische Gruppe mit dem Namen Mouvement d'Action et Défense Masada zu der Gewalt bekannte. Die aus einem neonazistischen Aktivenkreis um Michael Kühnen hervorgegangene Werwolfgruppe lenkte im Zusammenhang mit ihren Überfällen 1977/78 ,die Aufmerksamkeit auf Personen des linken Spektrums, indem sich die Täter als Linksextremisten ausgaben“ (Gräfe 2017, S. 126, unter Bezug auf das Urteil des OLG Celle). Der Rechtsterrorist Odfried Hepp sann mit Blick auf die von der Hepp/Kexel-Gruppe auf Angehörige der US-Streitkräfte verübten Bombenanschläge darüber nach, ob der Verdacht durch ein fingiertes Bekennerschreiben auf die Revolutionären Zellen gelenkt werden könne (ebd., S. 149f.), und Martin Wiese ließ im Zusammenhang mit dem geplanten Anschlag auf die Grundsteinlegung eines neuen jüdischen Kulturzentrums in München Drohbriefe in arabischer Sprache verfassen, damit der Eindruck entstünde, der Gewaltakt sei Teil des jüdisch-arabischen Konflikts (ebd., S. 185).

Umfangreichere Bekenntnisse setzen sich zu je unterschiedlichen Teilen aus weltanschaulich gestützten Begründungen, technischen und operativen Anleitungen, biographischen Bezügen und Handlungsappellen zusammen (Hartleb 2020). Insofern kommuniziert rechtsterroristische Gewalt in verschiedene Richtungen: an die politisch Nahestehenden, denen Handlungsbereitschaft und -mächtigkeit vermittelt werden soll, die ggfs. mit Rekrutierungsabsicht bzw. der Ermutigung zu Nachfolgetaten verbunden ist; an die soziale Gruppe, aus der stellvertretend Angehörige angegriffen werden, um diese einzuschüchtern, zu schädigen bzw. zu schwächen und um sie zu vertreiben; an staatliche Instanzen und politische Entscheider:innen, um diese unter Druck zu setzen.

\section{Rechtsterrorismus als Akt der Bestrafung, Einschüchterung und Vertreibung}

Der Anschlag auf die Synagoge in Halle/Saale am 9. Oktober 2019, dem höchsten jüdischen Feiertag Jom Kippur, hat im Judentum Erinnerungen an die Reichspogromnacht und die Shoah hervorgerufen (Weissberg 2020). Das vom Täter geplante 
Massaker an den Betenden wäre der bei weitem schwerste antisemitische und rechtsterroristische Anschlag in der Geschichte der beiden deutschen Staaten nach 1945 gewesen. Er hätte angesichts der vermutlich hohen Zahl an Getöteten und Verletzten weit über das jetzt zu konstatierende Ausmaß hinaus auch tiefsitzende Traumata von Verfolgungserfahrungen und antisemitischer Gewalt aktualisiert - und vermutlich bei erheblichen Teilen der jüdischen Bevölkerung den Gedanken in den Mittelpunkt gerückt, das Land zu verlassen. Manche erinnerte die Tat auch an Attentate der Federation National d'Action Europeene/Faiseaux Nationalistes Europeens, die Ende September 1980 in Paris mehrere jüdische Einrichtungen, darunter eine Synagoge, mit Maschinengewehren beschoss (Hoffman 1984, S. 3).

Rechtsterroristische Gewalt verfolgt das Ziel, die jeweils in konkreten Personen stellvertretend angegriffene soziale Gruppe zu verunsichern und in Angst zu versetzen sowie zum Rückzug aus dem öffentlichen Raum zu zwingen bzw. dazu, einen bestimmten Raum vollständig zu verlassen. Auch wenn es gezielte Morde an Menschen gibt, die einer breiteren Öffentlichkeit bekannt sind, so gilt vor allem: „Eben weil jedermann (innerhalb einer bestimmten Opfergruppe) zum Ziel werden kann, erhöht sich das Angstgefühl der Durchschnittspersonen innerhalb dieser Gruppen“ (Gräfe 2017, S. 247).

Das Freikorps Havelland, das zwischen August 2003 und Mai 2004 in Brandenburg sieben Brandanschläge auf Imbissstände beging, die von Unternehmern mit asiatischer oder türkischer Migrationsbiografie betrieben wurden, verzichtete auf Tatbekennungen. Durch die Zerstörung der ökonomischen Grundlage - manche Imbisse wurden mehrfach angezündet, weil die Täter sich erhofften, dass dann die Versicherung nicht mehr zahlen würde - sollten die Betreiber zum Fortzug genötigt werden. In Moskau verübten Mitglieder der rassistischen Gruppe Cnac (Der Erlöser) am 21. August 2006 einen Bombenanschlag auf den Tscherkisowoer Markt, dem 13 Menschen zum Opfer fielen. Die Gewalttat richtete sich gegen Händler:innen, die aus den asiatischen Republiken der früheren Sowjetunion dort ihre Stände betrieben. Auch die terroristische Gewalt der Deutschen Aktionsgruppen und des NSU zielte darauf $\mathrm{ab}$, über die unmittelbar von der Gewalt Betroffenen die Botschaften zu vermitteln, nicht willkommen zu sein, um Besitz und Leben fürchten zu müssen sowie schutzlos zu sein. Dies galt im Grundsatz auch für antiamerikanische Attentate auf US-Soldat:innen Anfang der 1980er-Jahre. Sie richteten sich - anders als bei der Roten Armee Fraktion - bewusst nicht gegen Führungspersonal, sondern gegen zufällig ausgewählte Soldat:innen, so dass dem Gros der Militärangehörigen vermittelt werden sollte, dass der deutsche Sicherheitsapparat wirkungslos sei.

Dem rechtsextremen Terrorismus der Bundesrepublik ging es immer wieder auch um die Einschüchterung und Schwächung der politischen Linken. Die Europäische Befreiungsfront (EBF) plante Sprengstoffanschläge auf Büros der Deutschen Kommunistischen Partei (DKP) und auf Einrichtungen der DGB-Gewerkschaften. Die Gruppe Stubbemann sah in einem Buchladen des Kommunistischen Bundes Westdeutschland (KBW) ein Ziel. Vom Attentat auf Rudi Dutschke am 11. April 1968 spannt sich der Bogen bis in die Gegenwart, etwa zur bereits mehrere Jahre anhaltenden Welle der Gewalt gegen Linke in Berlin-Neukölln. Teil dieser Gewaltpolitik ist die Sammlung von persönlichen Daten politischer Gegner:innen, die seit Jahrzehnten unter der Bezeichnung Anti-Antifa betrieben wird und als Grundlage von 
Anschlagsplanungen dient. Entsprechende Adresslisten fanden sich auch beim NSU und bei Breivik; sie stellten eine zentrale Grundlage der Auswahl der konkreten Anschlagsziele dar (Hemmingby und Bjørgo 2016).

In Sachsen setzten die Skinheads Sächsische Schweiz (SSS) seit 1996 ihren Handlungsschwerpunkt auf die Einschüchterung und Vertreibung von Personen, die von ihnen als ,links“ eingestuft wurden. Diese wurden zum Teil ,kurzzeitig gewaltsam fest[gehalten], um Fotos zu erstellen und andere Informationen zu erpressen“ (Gräfe 2017, S. 175). Die Daten wurden systematisch in einem digitalen ,Zeckenerfassungssystem' gespeichert. Auch der 2003 gegründete Sturm 34 fokussierte auf die politische Linke; dazu wurden planmäßig Treffpunkte angegriffen, in denen auch ,Punker“, ,Ökos ‘ und ,Linke“ verkehrten. Die späteren Opfer wurden dabei zum Teil im Zuge von so genannten ,Skinheadkontrollrunden` ausgewählt, bei denen Mitglieder des Sturm 34 in und um Mittweida mit PKW patrouillierten; die Gewaltpraxis war meist exzessiv (Backes et al. 2014) und führte dazu, dass in der Region Mittweida in den Jahren 2006 und 2007 Angehörige der linken Szene oder alternative Jugendliche nicht mehr öffentlich sichtbar waren. Bereits kursorische Blicke in andere Länder machen deutlich, dass terroristische Gewalt gegen die Linke zum Kern rechten Terrorismus gehört: Von den allein in Mailand im Jahr 1969 verübten 145 Anschlägen richtete sich die große Mehrheit gegen lokale Sektionen der Linksparteien sowie gegen Gewerkschaftsbüros (Hess 1988b, S. 54). In Finnland wurden in den 1970er-Jahren Briefbomben- und Brandanschläge auf Druckereien der dortigen Kommunistischen Partei, die Zentrale der Finnischen Demokratischen Jugendliga, die Botschaft Bulgariens sowie die Zeitung Kansan Uutiset verübt. In Spanien eskalierte rechtsterroristische Gewalt unmittelbar nach dem Ende der Franco-Diktatur; Gruppen wie Batallón Vasco Español, Guerrilleros de Cristo Rey oder Alianza Apostólica Anticommunista richteten ihre Gewalt insbesondere gegen Menschen, die der ETA zugerechnet wurden, sowie gegen die Linke. So wurden beim Atocha-Massaker am 24. Januar 1977 beispielweise fünf Mitglieder einer Gewerkschaft ermordet, die der Kommunistischen Partei nahestand. In den USA hat es zahlreiche Morde an Aktivist:innen der Bürgerrechtsbewegung gegeben; exemplarisch seien die von Mitgliedern verschiedener Ku Klux Klan-Gruppen verübten rassistischen Morde an Lemuel Penn (11. Juli 1964) und Viola Liuzzo (25. März 1965) genannt.

Rechtsterroristisches Handeln zielte in der Vergangenheit zudem auf die Einschüchterung von Journalist:innen sowie eine Störung der kritischen Aufarbeitung und Erinnerung an die Verbrechen des Nazismus. Entsprechend diskutierte die Werwolfgruppe die Sprengung eines Obelisken in der KZ-Gedenkstätte Bergen-Belsen; die beiden Neonazis Peter Naumann und Heinz Lembke führten 1978 in Italien einen Anschlag auf eine Gedenkstätte durch, die in der Nähe der Ardeatinischen Höhlen an die Ermordung von 335 zivilen italienischen Geiseln durch die WaffenSS im März 1944 erinnerte. Am 18. Januar 1979 verübten beide Sprengstoffanschläge auf Sendemasten, um die Ausstrahlung des vierteiligen Films Holocaust die Geschichte der Familie Weiss zu stören. In historischer Betrachtung markiert dieser Film den „Beginn der Bereitschaft nun auch eines Massenpublikums, sich mit der NS-Vergangenheit überhaupt auseinanderzusetzen“ (Reichel 2007, S. 261). Die Anschläge der Deutschen Aktionsgruppen auf das Landratsamt in Esslingen am 21. Februar 1980 bzw. das Wohnhaus des Landrates Hans Peter Braun am 18. April 
1980 zielten auf eine Ausstellung zum Vernichtungslager Auschwitz und verfolgten auch das Ziel der Einschüchterung. Schon am 2. September 1977 sowie am 21. Oktober 1977 hatte die Otte-Gruppe, benannt nach ihrem Anführer Paul Otte aus Braunschweig, Bombenanschläge auf die Staatsanwaltschaft Flensburg bzw. das Amtsgericht Hannover mit dem Ziel verübt, Strafprozesse gegen Rechtsextreme zu verhindern. Außerhalb Deutschlands verweist der Angriff von James Wenneker von Brunn auf das United States Holocaust Memorial Museum in Washington, DC, am 10. Juni 2009 auf entsprechende antisemitisch motivierte rechtsterroristische Taten.

Verschiedentlich kann rechtsterroristische Gewalt auch als Bestrafung bzw. Ausschaltung profilierter politischer Akteure verstanden werden. Dies gilt beispielweise für den Mord an Walther Lübcke, aber auch für die Ermordung des 78-jährigen Mahatma Gandhi am 30. Januar 1948 durch den fanatischen Hindu-Nationalisten Nathuram Godse, der als Mitglied der extrem rechten Akhil Bharatiya Hindu Mahasabha (Gesamtindische Hindu-Großversammlung) sowie der Rashtriya Swayamsevak Sangh (Nationale Freiwilligenorganisation) die politische Linie Gandhis entschieden ablehnte (Jaffrelot 1993; Delfs 2008). Die Ermordung des israelischen Ministerpräsidenten Jitzchak Rabin am 4. November 1995 durch den rechtsextremen, religiös-nationalistischen Jurastudenten Jigal Amir im Anschluss an eine Friedenskundgebung in Tel Aviv zielte auf die Schwächung bzw. Beendigung eines Verständigungsprozesses zwischen Israel, der palästinensischen Bevölkerung und arabischen Staaten, für den sich Rabin seit 1990 eingesetzt hatte (Kapeliuk 1997). Ein solch strafender Terrorismus versteht sich als Missbilligung und Sanktionierung von Normverletzungen und als expressiver Verweis auf den Geltungsanspruch spezifischer Normen, zu deren Aufrechterhaltung oder Durchsetzung er vielfach mit sich selbst droht - nicht zuletzt im Sinne präventiver Androhung von Strafe (Fischer 2014, S. 189, 192). In Einzelfällen richtet sich diese Gewalt auch gegen Angehörige des rechtsextremen Milieus; für sie wurde mit Blick auf eine entsprechende Gewaltpraxis in der Weimarer Republik der Begriff der Fememorde verwandt (Gumbel 1922). Entsprechende Fälle finden sich jedoch auch in den letzten Jahrzehnten; so etwa am 29. Mai 1981 der Mord an dem als homosexuell bekannten Mitglied der ANS, Johannes Bügner, in Hamburg, oder auch der Mord an Jonathan Bumstead, einem Mitglied der Aryan Nation, im September 1997, dem vorgeworfen wurde, jüdisch und ein ,race-traitor' zu sein.

Unter dem Gesichtspunkt der Bestrafung und Rache können auch die Attentate und Morde der sogenannten Incels (Kaiser 2020; Kracher 2020) betrachtet werden. Die heutige Incel-Subkultur wird dominiert von heterosexuellen Männern, die Frauenfeindlichkeit mit Gewaltphantasien propagieren; dabei verbindet sich ausgeprägt misogyne Hetze mit dem Anspruch, als Mann jederzeit Recht auf Sex mit Frauen $\mathrm{zu}$ haben. Aus der internationalen Incel-Subkultur sind inzwischen zahlreiche Morde begangen worden, so u.a. am 23. Mai 2014 durch Elliott Rodger (6 Tote, 13 Verletzte), der seinen privilegierten Anspruch auf Sex zudem mit seinem Weißsein begründete (Witt 2020; Kracher 2020, S. 63-88). Rodger wurde zum Idol von Nachfolgetätern wie Alek Minassian, der am 23. April 2018 in Toronto mit seinem Fahrzeug zehn Menschen tötete und sich zu Frauenhass als Tatmotiv bekannte. Auch Stefan Balliet, der am 9. Oktober 2019 ein Attentat auf die Synagoge und einen Döner-Imbiss in Halle/Saale beging, vertrat eine Incel-Ideologie. Darin 
wird feministischer Emanzipation eine zentrale Verantwortung für das eigene Leiden (Maxwell et al. 2020) zugeschrieben, das nach einer entsprechenden Reaktion verlange (Cottee 2021). Bis April 2020 sind durch Incel-Ideologie motivierte Gewalt fast 50 Menschen getötet worden (Hoffman et al. 2020; Tomkinson et al. 2020). Als Beta Male Uprising wird in Internet-Incel-Foren über einen gewaltsamen Aufstand gegen das System diskutiert, in dessen Verlauf eine möglichst große Zahl Frauen und sexuell aktive Menschen getötet werden sollen (Kaiser 2020); die Nachrichtenagentur NPR berichtete im Juli 2021 von der Festnahme eines Incel in Ohio, der mehrere Tausend Menschen töten wollte und sich zu diesem Zweck bewaffnet hatte (Diaz 2021; ceh 2021).

\section{Rechtsterrorismus als strategisches Moment der Eskalation}

Hess (1988a, S. 62) hat zwei grundlegende Strategien des Terrorismus unterschieden: Die eine orientiere sich auf die Einschränkung und Lähmung von Gruppen der Bevölkerung, die andere ziele auf den Umsturz. Nicht immer also richtet sich rechtsterroristische Gewalt gezielt gegen Menschen, die stellvertretend für eine bestimmte soziale Gruppe angegriffen werden. Das bedeutet gleichwohl nicht, dass sie ziellos ausgeübt würde. In Italien zwischen 1969 und 1984 von der Ordine Nuovo (Giannuli und Rosati 2017) und den vor ihr abgespaltenen Nuclei Armati Rivoluzionari verübte Terroranschläge, insbesondere das Bombenattentat auf der Piazza Fontana in Mailand am 12. Dezember 1969 (17 Tote, 88 Verletzte), der Anschlag von Gioia Tauro auf den Zug von Rom nach Messina am 22. Juli 1970 (6 Tote, 100 Verletzte), die Bombe im Bahnhof von Bologna am 2. August 1980 (85 Tote, mehr als 200 Verletzte) sowie ein Sprengsatz im italienischen Schnellzug 904 am 23. Dezember 1984 (27 Tote, 180 Verletzte), aber auch der Anschlag auf das Münchner Oktoberfest (Chaussy 1985, 2020) können als Versuche gewertet werden, gesellschaftliche Konflikte zuzuspitzen, ein Gefühl allgemeiner Unsicherheit hervorzurufen ${ }^{2}$ und auf diese Weise den Ruf nach autoritären Maßnahmen und einer Zentralisierung der staatlichen Exekutive zu befördern. Dabei wurde zum Teil versucht, diese Gewalttaten der politischen Linken anzulasten bzw. diese zu infiltrieren (Hess 1988b, S. 55).

Historisch lassen sich rechtsterroristische Gewalttaten als Mittel der strategischen Eskalation in Deutschland bereits in der Frühphase der Weimarer Republik identifizieren. So kommt der Historiker Martin Sabrow (1998) in seiner Rekonstruktion des Mordes an Außenminister Walther Rathenau zu dem Ergebnis, dass dieser Teil einer umfassenderen Planung der Organisation Consul war: Weil man in dieser antisemitischen terroristischen Gruppierung Rathenau als den zentralen Akteur der Regierung ansah, rechnete man als Folge seines Todes mit dem Sturz der Regierung und einer Zunahme linksradikaler Aktivitäten; als Reaktion war die Errichtung einer Militärdiktatur geplant.

\footnotetext{
2 „But even in the 1980 Bologna station attack and the bombing of the Italicus Express train (after four previous attempts) traveling through Bologna in 195, the indirect target was a city that is both strongly leftist and administered by a Communist Mayor“ (Hoffman 1984, S. 5).
} 
Die von mehreren rechtsterroristischen Gruppen in der Bundesrepublik Deutschland in den späten 1960er- und frühen 1970er-Jahren verübten oder geplanten Anschläge gegen Einrichtungen und Personal der DDR und der Sowjetunion waren nicht nur von antikommunistischem Hass getrieben, sondern hatten ebenfalls das Ziel, eine Verständigung und einen Modus Vivendi zwischen den beiden deutschen Staaten zu hintertreiben (Manthe 2020, S. 70). So plante etwa die 1970 um Roland Tabbert entstandene Nationale Deutsche Befreiungsbewegung (NDBB) mit Blick auf den zehnten Jahrestag des Mauerbaus Schüsse über die deutsch-deutsche Grenze sowie den Einsatz von Brandsätzen.

Der ,Tag X` - in den ersten Jahrzehnten der Bundesrepublik Deutschland seitens der extremen Rechten phantasiert als Tag des Einmarsches der Roten Armee und des Versuchs der Machtübernahme durch sozialistische und kommunistische Kader, seit 1980 zunehmend verknüpft mit der Vorstellung einer Eskalation sozialer Konflikte aufgrund zunehmender Immigration - ist ein Fixpunkt in den Strategieplanungen großer Teile der extremen Rechten und vielfach mit Vorstellungen bzw. Vorbereitungen zur Gewaltanwendung verbunden. Dies galt bereits früh für den Technischen Dienst (TD), eine Teilorganisation des am 23. Juni 1950 in Frankfurt am Main gegründeten antikommunistischen Bund Deutscher Jugend (BDJ) (Dudek und Jaschke 1984, S. 356-388). Im TD sammelten sich ehemalige Offiziere der Wehrmacht und der Waffen-SS für den Fall eines befürchteten Einmarsches der sowjetischen Roten Armee, dem man mit einer in Partisanenkriegführung geschulten Einheit entgegentreten wollte. Der BDJ-Gründer Paul Lüth führte zudem in seiner Schrift Bürger und Partisan aus, dass angesichts der ,kommunistischen Infiltration' schon in Friedenszeiten gegen die politische und gewerkschaftliche Linke vorgegangen werden müsse (Lüth 1951). Ein eigener Nachrichtendienst sammelte Informationen über politische Gegner:innen, meist hochrangige SPD-Politiker, die am ,Tag X kaltgestellt' bzw. ,aus dem Verkehr gezogen' werden sollten - so entstand eine Liste mit den Namen von vierzig Personen (Hessischer Minister für Inneres 1954).

In Krisenzeiten verschärft sich die extrem rechter Wirklichkeitswahrnehmung zugrundeliegende kulturpessimistische Vorstellung vom Niedergang der ,weißen“ Gesellschaften aufgrund von Einwanderung und gesellschaftlicher Liberalisierung, die im Detail je nach nationalstaatlichem und historischem Kontext variiert (SPLC 2009), zu Bildern einer bevorstehenden Apokalypse und millenialistischen Szenarien. Zugleich finden sich auch Erwartungen und Hoffnungen, dass der ,Volkstod“ in dem erwarteten Bürgerkrieg noch einmal abgewandt werden könne. Auf solche Konstellationen, die ebenfalls mit der Vorstellung des ,Tag $\mathrm{X}^{`}$ verbunden werden, bereitet sich eine wachsende Zahl von Gruppen und Netzwerken vor, wie beispielsweise die Ermittlungen zu Gruppen wie Nordkreuz ergeben haben (Laabs 2021).

Einzelne rechtsterroristische Gruppen zielen mit ihren Gewalttaten darauf ab, den prognostizierten gesellschaftlichen Zusammenbruch durch die von ihnen verübten Gewalttaten zu beschleunigen (akzelerationistischer Terrorismus). Dies gilt etwa für die in den USA seit 2015 sichtbare Atomwaffen-Division (Ware 2019; Soufan Center 2020), aber auch für Gruppen wie die Oldschool Society und Revolution Chemnitz. Der seit Mitte April 2021 vor dem OLG Stuttgart angeklagten Gruppe S. wird ebenfalls vorgeworfen, sie habe mittels zeitgleich durchzuführender Anschläge 
auf mehrere Moscheen Unruhen und Gewalt seitens gläubiger Muslime hervorrufen wollen.

\section{Fazit}

Rechtsterroristische Akteur:innen rechtfertigen die Gewalt mit einer Erzählung von Notwehr und subjektivem Leiden sowie der zur Rettung von Volk, Nation und "Rasse' als unausweichlich behaupteten Konfrontation. Diese ,moralistic violence“ (Michalski 2019) behauptet Normverletzungen im Sinne der Verletzung von ,Reinheitsnormen', die in der Benachteiligung der Eigengruppe sowie der Nichtbeachtung angeblich naturgegebener Hierarchien und Superioritätsansprüche wirksam würden (Fischer 2014, S. 188; VandenBerg 2021). Das Narrativ von der Verteidigung der ,weißen Rasse" wird dabei ebenso als Legitimation aufgerufen wie die Beschwörung der Gefahren eines ,Kultur-Marxismus“ (Busbridge et al. 2020). Die Bandbreite rechtsterroristischer Handlungsmodi und Organisationsstrukturen ist ebenso groß wie die potenziellen Zielgruppen entsprechend den nationalen Kontexten und historischen Konstellationen vielfältig. Dieser Terror setzt Gewalt instrumentell ein, um Hegemonie im Sozialraum herzustellen, um soziale Gruppen, aber auch konkrete Individuen einzuschüchtern, zu schädigen und zu vertreiben, um eigene Handlungsmächtigkeit zu demonstrieren ${ }^{3}$ und die angestrebte soziale Ordnung tendenziell herzustellen sowie schließlich auch, um die als, Volksfeinde“ bezeichneten Menschen tatsächlich zu dezimieren. In welchem Maße die beabsichtigte Wirkung bei den Angehörigen der sozialen Gruppe, der die jeweilige Gewalttat gilt, tatsächlich eintritt, ob das Vertrauen in staatliche Institutionen und Handlungspraxen in Folge einer rechtsterroristischen Gewalttat beeinträchtigt wird (Satherley et al. 2021), oder ob sich nach solchen Gewalttaten die Einstellung in der Bevölkerung zu kontroversen politischen Fragen ändert (Jose et al. 2021), hängt von zahlreichen Faktoren ab. Hierzu zählen etwa die mediale Rahmung der Gewalttat (Hancock und Haldeman 2017; Walter et al. 2017), der Grad der gesellschaftlichen Exklusion und Diskriminierung der sozialen Gruppe, die angegriffen wurde (Stults et al. 2017), aber auch die Reaktionen der betroffenen sozialen Gruppe (Jackson 2017; Jenkins et al. 2019).

Der Rechtsterrorismus ist zeitlich und räumlich in sehr unterschiedlichen Formen und Strukturen in Erscheinung getreten: als bewaffnete Formationen, die gezielt als Feinde markierte Menschen ermordeten, als kleine Gruppen, die - unterstützt von einem Netzwerk von Sympathisant:innen - über Jahre hinweg heimlich mordeten, aber auch als Scharfschützen und so genannte Einzeltäter. Sie verstanden sich oft als Avantgarde, was sie durch die schiere Brutalität ihrer Taten und die Botschaften, die sie damit vermitteln wollten, zum Ausdruck brachten. In jüngster Zeit werden solche Terrorakte in der Regel einem internationalen Publikum mitgeteilt, wenn möglich sogar per Live-Streaming. In Manifesten, die auf Online-Plattformen veröffentlicht wurden, wurden die ideologischen Begründungen und taktischen Überlegungen dargelegt und Hinweise für die Beschaffung und Herstellung von Waffen geliefert. Die

\footnotetext{
${ }^{3} \mathrm{Zu}$ erinnern ist an zahlreiche Pläne rechtsterroristischer Gruppen, Rudolf $\mathrm{He} ß$ aus dem Spandauer Kriegsverbrecher-Gefängnis zu befreien.
} 
Versuche, transnationale rechtsterroristische Strukturen wie die Atomwaffendivision und ähnliche Gruppen aufzubauen, nehmen zu, aber die operative Basis der rechtsterroristischen Gruppen bleibt in den meisten Fällen an einen bestimmten Nationalstaat gebunden.

Funding Open Access funding enabled and organized by Projekt DEAL.

Open Access Dieser Artikel wird unter der Creative Commons Namensnennung 4.0 International Lizenz veröffentlicht, welche die Nutzung, Vervielfältigung, Bearbeitung, Verbreitung und Wiedergabe in jeglichem Medium und Format erlaubt, sofern Sie den/die ursprünglichen Autor(en) und die Quelle ordnungsgemäß nennen, einen Link zur Creative Commons Lizenz beifügen und angeben, ob Änderungen vorgenommen wurden.

Die in diesem Artikel enthaltenen Bilder und sonstiges Drittmaterial unterliegen ebenfalls der genannten Creative Commons Lizenz, sofern sich aus der Abbildungslegende nichts anderes ergibt. Sofern das betreffende Material nicht unter der genannten Creative Commons Lizenz steht und die betreffende Handlung nicht nach gesetzlichen Vorschriften erlaubt ist, ist für die oben aufgeführten Weiterverwendungen des Materials die Einwilligung des jeweiligen Rechteinhabers einzuholen.

Weitere Details zur Lizenz entnehmen Sie bitte der Lizenzinformation auf http://creativecommons.org/ licenses/by/4.0/deed.de.

\section{Literatur}

\section{Verwendete Literatur}

Backes, Uwe, Anna-Maria Haase, Michail Logvinov, Matthias Mletzko, und Jan Stoye. 2014. Rechts motivierte Mehrfach- und Intensivtäter in Sachsen. Göttingen: V \& R unipress.

Baird-Windle, Patricia, und Eleanor J. Bader. 2001. Targets of hatred. Anti-abortion terrorism. New York: Palgrave.

Billstein, Thomas. 2020. Kein Vergessen. Todesopfer rechter Gewalt in Deutschland nach 1945. Münster: Unrast.

Bott, Hermann. 1969. Die Volksfeindideologie. Zur Kritik rechtsradikaler Propaganda. Stuttgart: DVA.

Bozay, Kemal, Bahar Aslan, Orhan Mangitay, und Funda Özfirat. 2016. Die haben gedacht, wir waren das - MigrantInnen über rechten Terror und Rassismus. Köln: PapyRossa.

Bozay, Kemal, Serpil Güner, Orhan Mangitay, und Funda Göçer (Hrsg.). 2021. Damit wir atmen können. Migrantische Stimmen zu Rassismus, rassistischer Gewalt und Gegenwehr. Köln: PapyRossa.

Brown, David. 2020. Knifeman 'attacked law firm that had helped migrants'. The Times vom 20. Oktober 2020.

Busbridge, Rachel, Benjamin Moffitt, und Joshua Thorburn. 2020. Cultural Marxism: far-right conspiracy theory in Australia's culture wars. Social Identities 26(6):722-738.

Caneva, Augusto. 1986. Il caso Ludwig. Trient: Luigi Reverdito Editore.

ceh. 2021. Mann plante Massenmord an Frauen. Frankfurter Allgemeine Zeitung vom 23. Juli 2021.

Chaussy, Ulrich. 1985. Oktoberfest. Ein Attentat. Darmstadt: Luchterhand.

Chaussy, Ulrich. 2020. Das Oktoberfest-Attentat und der Doppelmord von Erlangen: Wie Rechtsterrorismus und Antisemitismus seit 1980 verdrängt werden. Berlin: Ch. Links.

Cottee, Simon. 2021. Incel (E)motives: resentment, shame and revenge. Studies in Conflict \& Terrorism 44(2):93-114.

Delfs, Tobias. 2008. Hindu-Nationalismus und europäischer Faschismus. Vergleich, Transfer- und Beziehungsgeschichte. Hamburg-Schenefeld: EB-Verlag.

Della Porta, Donatella. 2013. Clandestine political violence. Cambridge: Cambridge University Press.

Diaz, Jaclyn. 2021. Police Foiled An Ohio Incel's Plot To Kill Women In A Mass Shooting, Prosecutors Say. NPR vom 22. Juli 2021. https://www.npr.org/2021/07/22/1019089834/police-foiled-an-ohioincels-plot-to-kill-women-in-a-mass-shooting-prosecutors-s?t=1628019103420. Zugegriffen: 4. Okt. 2021. 
Dudek, Peter, und Hans-Gerd Jaschke. 1984. Entstehung und Entwicklung des Rechtsextremismus in der Bundesrepublik. Bd. 1. Opladen: Westdeutscher Verlag.

Dunning, Michael. 2021. Britain and terrorism. A sociological investigation. Cham: Palgrave Macmillan. Fischer, Michael. 2014. Terrorismus und staatliches Handeln: Figurationen der sozialen Kontrolle. In Die Sinnprovinz der Kriminalität, Hrsg. Henning Schmidt-Semisch, Henner Hess, 187-202. Wiesbaden: Springer VS.

Foucault, Michel. 1983. Sexualität und Wahrheit 1: Der Wille zum Wissen. Frankfurt am Main: Suhrkamp.

Foucault, Michel. 1999. Verteidigung der Gesellschaft. Frankfurt am Main: Suhrkamp. Vorlesungen am Collège de France (1975/1976).

Foucault, Michel. 2004. Geschichte der Gouvernementalität. Die Geburt der Biopolitik. Frankfurt am Main: Suhrkamp. Vorlesungen am Collège de France, 1978-1979.

Fuchs, Peter. 2004. Das System ,Terror‘. Versuch über eine kommunikative Eskalation der Moderne. Bielefeld: transcript.

Giannuli, Aldo, und Elia Rosati. 2017. Storia di Ordine Nuovo. Mailand: Mimesis.

Gräfe, Sebastian. 2017. Rechtsterrorismus in der Bundesrepublik. Zwischen erlebnisorientierten Jugendlichen, ,Feierabendterroristen ‘ und klandestinen Untergrundzellen. Baden-Baden: Nomos.

Gullestad, Siri Erika. 2017. Anders Behring Breivik, master of life and death: Psychodynamics and political ideology in an act of terrorism. International Forum of Psychoanalysis 26(4):207-216.

Gumbel, Emil Julius. 1922. Vier Jahre politischer Mord. Berlin: Verlag der Neuen Gesellschaft.

Hancock, Kristin A., und Douglas C. Haldeman. 2017. Between the lines: media coverage of Orlando and beyond. Psychology of Sexual Orientation and Gender Diversity 4(2):152-159.

Hartleb, Florian. 2020. Die Manifeste rechtsterroristischer Einzeltäter: Eine vergleichende Analyse. Kriminalistik 72(5):313-318.

Hemmingby, Cato, und Tore Bjørgo. 2016. The dynamics of a terrorist targeting process. Anders B. Breivik and the 22 july attacks in Norway. New York: Palgrave macmillan.

Hess, Henner. 1988a. Terrorismus und Terrorismus-Diskurs. In Angriff auf das Herz des Staates. Soziale Entwicklung und Terrorismus, Bd. 1, Hrsg. Henner Hess, Martin Moerings, Dieter Paas, Sebastian Scheerer, und Heinz Steinert, 55-74. Frankfurt am Main: Suhrkamp.

Hess, Henner. 1988b. Italien. Die ambivalente Revolte. In Angriff auf das Herz des Staates. Soziale Entwicklung und Terrorismus, Bd. 2, Hrsg. Henner Hess, Martin Moerings, Dieter Paas, Sebastian Scheerer, und Heinz Steinert, 9-166. Frankfurt am Main: Suhrkamp.

Hoffman, Bruce. 1984. Right-wing terrorism in Europe since 1980. Rand paper series P-7029. Santa Monica: The Rand Corporation.

Hoffman, Bruce, Jacob Ware, und Ezra Shapiro. 2020. Assessing the Threat of Incel Violence. Studies in Conflict \& Terrorism 43(7):565-587.

Jackson, Paul. 2013. The license to hate: Peder Jensen's fascist rhetoric in Anders Breivik's manifesto 2083: a European declaration of independence. Democracy and Security 9(3):247-269.

Jackson, Skyler D. 2017. 'Connection is the antidote': psychological distress, emotional processing, and virtual community building among LGBTQ students after the Orlando shooting. Psychology of Sexual Orientation and Gender Diversity 4(2):160-168.

Jaffrelot, Christophe. 1993. The Hindu nationalist movement and Indian politics. London: Hurst \& Co.

Jenkins, Philip. 1988. Under two flags: provocation and deception in European terrorism. Terrorism 11(4):275-287.

Jenkins, Elizabeth M., Zulfia Zaher, Stephanie A. Tikkanen, und Jessica L. Ford. 2019. Creative identity (re)construction, creative community building, and creative resistance: a qualitative analysis of queer ingroup members' tweets after the Orlando Shooting. Computers in Human Behavior 101:14-21.

John, Barbara (Hrsg.). 2014. Unsere Wunden kann die Zeit nicht heilen - Was der NSU-Terror für die Opfer und Angehörigen bedeutet. Freiburg: Herder.

Jose, Rupa, E. Alison Holman, und Roxane Silver Cohen. 2021. How americans feel about guns after mass shootings: the case of the 2016 Orlando nightclub massacre. Psychology of Violence 11(4):354-363.

Kaiser, Susanne. 2020. Politische Männlichkeit. Wie Incels, Fundamentalisten und Autoritäre für das Patriarchat mobilisieren. Berlin: Suhrkamp.

Kapeliuk, Amnon. 1997. Rabin. Ein politischer Mord. Nationalismus und rechte Gewalt in Israel. Heidelberg: Palmyra.

Kearns, Erin M., Brendan Conlon, und Joseph K. Young. 2014. Lying about Terrorism. Studies in Conflict \& Terrorism 37(5):422-439.

Knoll, Albert (Hrsg.). 2020. Der Anschlag auf Magnus Hirschfeld. Ein Blick auf das reaktionäre München 1920. München: Forum Queeres München. 
Koehler, Daniel. 2016. Right-wing terrorism in the 21th century. The 'national socialist underground' and the history of terror from the far right in Germany. Florence: Taylor \& Francis.

Kracher, Veronika. 2020. Incels. Geschichte, Sprache und Ideologie eines Online-Kults. Mainz: Ventil.

Laabs, Dirk. 2021. Staatsfeinde in Uniform. Berlin: Econ.

Landtag Nordrhein-Westfalen. 2017. Schlussbericht des Parlamentarischen Untersuchungsausschusses III. Drucksache 16/14400. Düsseldorf: Landtag Nordrhein-Westfalen.

Lemke, Thomas. 2008. Eine Analytik der Biopolitik. Überlegungen zur Geschichte und Gegenwart eines umstrittenen Begriffs. Behemoth. A Journal of Civilisation 1(1):72-89.

Lüttig, Frank, und Jens Lehmann (Hrsg.). 2020. Rechtsextremismus und Rechtsterrorismus. Baden-Baden: Nomos.

Manthe, Barbara. 2020. Rechtsterroristische Gewalt in den 1970er Jahren. Die Kühnen-Schulte-WegenerGruppe und der Bückeburger Prozess 1979. Vierteljahrshefte für Zeitgeschichte 1:63-94.

Maxwell, December, Sarah H. Robinson, Jessica R. Williams, und Craig Keaton. 2020. 'A short history of a lonely guy': a qualitative thematic analysis of involuntary celibacy using reddit. Sexuality \& Culture 24(4):1852-1874.

Michalski, Joseph H. 2019. Terrorism and lethal moralism in the United States and United Kingdom, 1970-2017. The British Journal of Sociology 70(5):1681-1708.

Nossek, Hillel. 2008. 'News media'-media events. Terrorist acts as media events. Communications 33(3):313-330.

Philippsberg, Robert. 2021. Rechtsterroristische Gruppen nach dem NSU. Zeitschrift für Rechtsextremismusforschung 1:147-166.

Pörksen, Bernhard. 2000. Die Konstruktion von Feindbildern. Zum Sprachgebrauch in neonazistischen Medien. Wiesbaden: Westdeutscher Verlag.

Post, Jerald M., Cody McGinnis, und Kristen Moody. 2014. The changing face of terrorism in the 21th century: the communications revolution and the virtual community of hatred. Behavioral Sciences and the Law 3:306-334.

Quent, Matthias. 2019. Rassismus, Radikalisierung, Rechtsterrorismus. Wie der NSU entstand und was er über die Gesellschaft verrät. Weinheim: BeltzJuventa.

Reichel, Peter. 2007. Erfundene Erinnerung - Weltkrieg und Judenmord in Film und Theater. Frankfurt am Main: Fischer.

Rothenberger, Liane. 2020. Terrorismus als Kommunikation. Bestandsaufnahme, Erklärungen und Herausforderungen. Wiesbaden: VS.

Sabrow, Martin. 1998. Die verdrängte Verschwörung. Der Rathenau-Mord und die deutsche Gegenrevolution. Frankfurt am Main: Fischer.

Sandberg, Sveinung. 2013. Are self-narratives strategic or determined, unified or fragmented? Reading Breivik's Manifesto in light of narrative criminology. Acta Sociologica 56(1):69-83.

Sarasin, Philipp, und Dieter Thomä. 2008. Biopolitik. In Handbuch der politischen Philosophie und Sozialphilosophie, Hrsg. Stefan Gosepath, Wilfried Hinsch, und Beate Rössler, 147-152. Berlin: De Gruyter.

Satherley, Nicole, Kumar Yogeeswaran, Danny Osborne, Sadi Shanaah, und Chris G. Sibley. 2021. Investigating the effects of right-wing terrorism on government satisfaction: a time course analysis of the 2019 Christchurch terror attack. Studies in Conflict \& Terrorism :1-20.

Schmid, Alex P., und Janny de Graaf. 1982. Violence as communication. Insurgent terrorism and the western news media. London, Beverly Hills: SAGE.

Schultz, Tanjev_PARTICLEDOT_. 2018. NSU - Der Terror von rechts und das Versagen des Staates. München: Droemer.

Şimşek, Semiya. 2013. Schmerzliche Heimat - Deutschland und der Mord an meinem Vater. Berlin: Rowohlt.

Soufan Center. 2020. The Atomwaffen division. The evolution of the white supremacy threat. New York: Soufan Center.

SPLC/Southern Poverty Law Center. 2009. Terror from the right. 75 plots, conspiracies and racist rampages since oklahoma city. Montgomery: SPLC.

Steinhagen, Martin. 2021. Rechter Terror. Der Mord an Walter Lübcke und die Strategie der Gewalt. Hamburg: Rowohlt.

Steinke, Ronen. 2020. Terror gegen Juden. Wie antisemitische Gewalt erstarkt und der Staat versagt. Berlin: Berlin Verlag.

Strohmaier, Jürgen. 1982. Manfred Roeder - ein Brandstifter. Dokumente und Hintergründe zum Stammheimer Neofaschisten-Prozeß. Stuttgart: Selbstverlag. 
Stults, Christopher B., Sandra A. Kupprat, Kristen D. Krause, Farzana Kapadia, und Perry N. Halkitis. 2017. Perceptions of safety among LGBTQ people following the 2016 pulse nightclub shooting. Psychology of Sexual Orientation and Gender Diversity 4(3):251-256.

Tomkinson, Sian, Harper Tauel, und Katie Attwell. 2020. Confronting Incel: exploring possible policy responses to misogynistic violent extremism. Australian Journal of Political Science 55(2):152-169.

Tuman, Joseph S. 2010. Communicating terror. The rhetorical dimensions of terrorism. Thousand Oaks: SAGE.

Turk, Austin T. 2004. Sociology of terrorism. Annual Review of Sociology 30:271-286.

Unterholzner, Bernhard. 2007. Bekennerschreiben. Kommunikation als Ereignis. Saarbrücken: VDM.

VandenBerg, Robert J. 2021. Legitimating extremism: a taxonomy of justifications for political violence. Terrorism and Political Violence 33(6):1237-1255.

Virchow, Fabian. 2016. Nicht nur der NSU - eine kleine Geschichte des Rechtsterrorismus in Deutschland. Erfurt: LpB.

Virchow, Fabian, und Tanja Thomas. 2021. Doing Memory an rechte Gewalt in Medienkulturen. Grundzüge eines interdisziplinären Forschungsprogramms. In Rechte Gewalt erzählen. Doing Memory in Literatur, Theater und Film, Hrsg. Matthias N. Lorenz, Tanja Thomas, und Fabian Virchow. Heidelberg: J.B. Metzler.

Virchow, Fabian, Tanja Thomas, und Elke Grittmann. 2015. Das Unwort erklärt die Untat‘. Die Berichterstattung über die NSU-Morde. Eine Medienkritik. Frankfurt am Main: OBS.

Waldmann, Peter. 2005. Terrorismus. Provokation der Macht. Hamburg: Murmann.

Walter, Nathan, Thomas J. Billard, und Sheila T. Murphy. 2017. On the boundaries of framing terrorism: guilt, victimization, and the 2016 Orlando shooting. Mass Communication and Society 20(6):849-868.

Ware, Jacob. 2019. Siege: the Atomwaffen division and rising far-right terrorism in the United States. The Hague: ICCT.

Weissberg, Nea (Hrsg.). 2020. Halle ist überall. Stimmen jüdischer Frauen. Berlin: Lichtig.

Wilson, Yoshua C. 2013. The street politics of abortion. Speech, violence, and America's culture wars. Stanford: Stanford University Press.

Wilson, Janet. 2014. Toxic rhetoric: the language of the turner diaries: a novel. In Doublespeak. The rhetoric of the far right since 1945, Hrsg. Matthew Feldman, Paul Jackson, 61-80. Stuttgart: ibidem.

Witt, Taisto. 2020. 'If i cannot have it, i will do everything i can to destroy it.' The canonization of Elliot Rodger: 'Incel' masculinities, secular sainthood, and justifications of ideological violence. Social Identities 26(5):675-689.

Wojciechowski, Sebastian. 2017. Reasons of contemporary terrorism. An analysis of main determinants. In Radicalism and terrorism in the 21st century, Hrsg. Anna Sroka, Fanny Castro-Rial Garrone, und Rubén Darío Torres Kumbrián, 49-70. Frankfurt und Bern: Peter Lang.

Zornetta, Monica. 2011. Ludwig. Storie du fuoco, sangue, follia. Mailand: Baldini Castoldi Dalai.

\section{Quellenverzeichnis}

Hessischer Minister für Inneres. 1954. Der Technische Dienst des Bundes Deutscher Jugend (BDJ). Wiesbaden: Hessischer Minister für Inneres. 3 Bände.

Kjellén, Rudolf. 1920. Grundriß zu einem System der Politik. Leipzig: Hirzel.

Lüth, Paul. 1951. Bürger und Partisan. Über den Widerstand gestern, heute und morgen. Frankfurt am Main: Parma.

NPD. 2015. 5-Punkte-Plan zur Ausländerrückführung. Berlin: Hessischer Minister für Inneres.

Peterson, Steven A., und Albert Somit (Hrsg.). 2017. Handbook of Biology and Politics. Cheltenham: Edward Elgar Publishing.

\section{Weiterführende Literatur}

Byman, Daniel. 2019. The threat from within. The National Interest 162:52-62.

Kleffner, Heike, und Matthias Meisner (Hrsg.). 2019. Extreme Sicherheit. Rechtsradikale in Polizei, Verfassungsschutz, Bundeswehr und Justiz. Freiburg/Br: Herder. 